Louisiana State University

LSU Digital Commons

$1-1-1999$

\title{
Spatiotemporal separation of high harmonic radiation into two quantum path components
}

\author{
M. B. Gaarde \\ Lunds Tekniska Högskola \\ F. Salin \\ Université de Bordeaux \\ E. Constant \\ Université de Bordeaux \\ $\mathrm{Ph}$ Balcou \\ CNRS Centre National de la Recherche Scientifique \\ K. J. Schafer \\ Louisiana State University
}

See next page for additional authors

Follow this and additional works at: https://digitalcommons.Isu.edu/physics_astronomy_pubs

\section{Recommended Citation}

Gaarde, M., Salin, F., Constant, E., Balcou, P., Schafer, K., Kulander, K., \& L'Huillier, A. (1999). Spatiotemporal separation of high harmonic radiation into two quantum path components. Physical Review A - Atomic, Molecular, and Optical Physics, 59 (2), 1367-1373. https://doi.org/10.1103/PhysRevA.59.1367 


\section{Authors}

M. B. Gaarde, F. Salin, E. Constant, Ph Balcou, K. J. Schafer, K. C. Kulander, and A. L'Huillier 


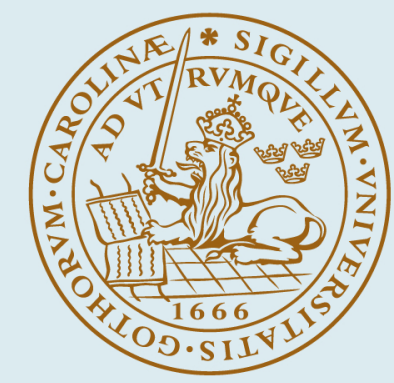

\title{
LUND UNIVERSITY
}

\section{Spatiotemporal separation of high harmonic radiation into two quantum path components}

\author{
Gaarde, Mette; Salin, F; Constant, E; Balcou, P; Schafer, K. J; Kulander, K. C; L'Huillier, Anne
}

Published in:

Physical Review A (Atomic, Molecular and Optical Physics)

DOI:

10.1103/PhysRevA.59.1367

1999

Link to publication

Citation for published version (APA):

Gaarde, M., Salin, F., Constant, E., Balcou, P., Schafer, K. J., Kulander, K. C., \& L'Huillier, A. (1999).

Spatiotemporal separation of high harmonic radiation into two quantum path components. Physical Review $A$ (Atomic, Molecular and Optical Physics), 59(2), 1367-1373. https://doi.org/10.1103/PhysRevA.59.1367

Total number of authors:

7

\footnotetext{
General rights

Unless other specific re-use rights are stated the following general rights apply:

Copyright and moral rights for the publications made accessible in the public portal are retained by the authors

and/or other copyright owners and it is a condition of accessing publications that users recognise and abide by the legal requirements associated with these rights.

- Users may download and print one copy of any publication from the public portal for the purpose of private study or research.

- You may not further distribute the material or use it for any profit-making activity or commercial gain

- You may freely distribute the URL identifying the publication in the public portal

Read more about Creative commons licenses: https://creativecommons.org/licenses/

Take down policy

If you believe that this document breaches copyright please contact us providing details, and we will remove access to the work immediately and investigate your claim.
} 


\title{
Spatiotemporal separation of high harmonic radiation into two quantum path components
}

\author{
M. B. Gaarde, ${ }^{1,2}$ F. Salin, ${ }^{3}$ E. Constant,${ }^{3}$ Ph. Balcou, ${ }^{4}$ K. J. Schafer,${ }^{5}$ K. C. Kulander, ${ }^{6}$ and A. L'Huillier ${ }^{1}$ \\ ${ }^{1}$ Department of Physics, Lund Institute of Technology, P.O. Box 118, S-22100 Lund, Sweden \\ ${ }^{2}$ Niels Bohr Institute, Orsted Laboratory, Universitetsparken 5, 2100 Copenhagen D, Denmark \\ ${ }^{3}$ CPMOH/ELIA, Université Bordeaux 1, 351 Cours de la Libération, 33405 Talence Cedex, France \\ ${ }^{4}$ Laboratoire d'Optique Appliquée, ENSTA, Ecole Polytechnique, Unité Mixte de Recherches 7639, \\ Centre National de la Recherche Scientifique, 91761 Palaiseau Cedex, France \\ ${ }^{5}$ Department of Physics and Astronomy, Louisiana State University, Baton Rouge, Louisiana 70803-4001 \\ ${ }^{6}$ TAMP Group, Lawrence Livermore National Laboratory, Livermore, California 94551
}

(Received 4 August 1998)

\begin{abstract}
We present a spatiotemporal analysis of high harmonic generation, showing evidence for the presence of several quantum path contributions to the atomic dipole moment. We show that he harmonic radiation can largely be described as a sum of two fields having a phase proportional to the intensity of the generating field. We compare our results to recent experimental results demonstrating this separation [M. Bellini et al., Phys. Rev. Lett. 81, 297 (1998)]. We show how the temporal and spatial coherence properties are influenced by this effect, and discuss how it could be used to obtain better control of the generated harmonic radiation. [S1050-2947(99)00402-3]

PACS number(s): $32.80 . \mathrm{Rm}, 42.65 . \mathrm{Ky}$
\end{abstract}

\section{INTRODUCTION}

Since the first observations of high harmonic generation about a decade ago, much effort has been put into systematic studies of this phenomenon, both experimental and theoretical [2]. The harmonic radiation-generated in the interaction between a very strong laser field and free atoms-has recently been rather well characterized; the pulse length has been measured [3], and the temporal $[1,4,5]$ and spatial [6] coherence properties have been studied. The harmonic radiation presents a unique source in the extreme ultraviolet (XUV) spectral region, due to its table-top size and its very short pulse length, and the interest in using this radiation for applications is growing. For some of these applications, the characterization and optimization of the spatial and temporal coherence of the harmonic radiation is very important.

The understanding of high-order harmonic generation processes has progressed considerably during the past 5 years, largely owing to the development of the semiclassical interpretation $[7,8]$. In this approach, an electron initially in the ground state of an atom, and exposed to an intense, lowfrequency, linearly polarized, electromagnetic field, first tunnels through the barrier formed by the Coulomb and the laser fields. When the laser field changes sign, the electron may be driven back towards the core with high kinetic energy and recombine to the ground state, giving rise to emission of high-energy harmonic photons. A quantum-mechanical formulation of this model, known as the strong field approximation (SFA) $[9,10]$, has been used extensively to describe different aspects of the generation process.

The SFA predicts that, for a harmonic belonging to the plateau region, there are several electronic trajectories that contribute to the generation process, giving rise to different, interfering, contributions to the dipole moment of some particular harmonic. An electronic trajectory-or quantum path-is characterized by the time of tunneling, and the time it spends in the continuum, called the return time $\tau$. Mainly two of these trajectories play a dominant role: One has a return time $\tau_{2}$ very close to a full period of the laser field, whereas the other has a shorter return time $\tau_{1}$, less than half a laser period [11]. For a harmonic belonging to the cutoff region, there is only one quantum path contributing to the generation process, with a return time of approximately half a laser period. The phase of the emitted radiation is locked to the phase of the electronic wave packet in the continuum, and the different quantum paths, therefore, contribute with different phase behavior to the dipole moment. The $\tau_{1}$ path contributes with a phase that varies linearly and quite slowly as a function of the laser intensity, whereas the phase of the $\tau_{2}$ quantum path decreases linearly and rapidly with the intensity.

In a recent experiment performed by Bellini et al. [1], measuring the temporal coherence of the harmonics 7-21 in argon, the presence of the two above-mentioned contributions to the atomic dipole moment was demonstrated. This was seen as a separation of the far-field profile of the 15th harmonic into two spatial regions having different temporal coherence times: An inner region with a long coherence time, interpreted as being due to the $\tau_{1}$ contribution, and an outer region, with a much shorter coherence time, due to the $\tau_{2}$ contribution. In the present paper we extend the theoretical analysis presented in [1], and use a simple description of harmonic generation-based on the SFA - to interpret the observed experimental results. We present a spatiotemporal analysis of the harmonic radiation in terms of the two contributing quantum paths, showing how these can be separated both in the space and time domain. We compare the results of the simple model to results of more elaborate numerical calculations, using data calculated by numerical integration of the time-dependent Schrödinger equation [12].

The paper is organized as follows: In Sec. II we present a simple model, describing the harmonic radiation as a sum of two fields, each with a phase proportional to the intensity of the driving field. From simple arguments, we predict surpris- 
ingly well the behavior of the macroscopic harmonic field. In Sec. III we present more elaborate numerical calculations, along with some numerical results, and compare these to experimental results from the literature $[1,13,14]$. Finally, in Sec. IV we discuss our results, and suggest means of controlling the coherence properties of the generated radiation.

\section{MODEL DESCRIPTION}

From the above discussion of the different quantum path contributions to the atomic dipole moment, we assume that the harmonic field at the frequency $q \omega_{0}$ consists of two components, $i=1,2$, each with a phase dependence

$$
\Phi_{i}(r, z, t)=-\alpha_{i} I(r, z, t) .
$$

Here $\omega_{0}$ is the laser frequency, $I(r, z, t)$ is the space- and time-dependent intensity of the generating field, and $\alpha_{i}$ is the slope of the phase as function of intensity for each of the two quantum path components. We use cylindrical coordinates.

The intensity dependence of the phase in Eq. (1) is very important. In a real experiment, one uses a pulsed, and focused, laser beam, characterized by an intensity $I(r, z, t)$. The variation in time of the intensity, $I(t)$, induces a change in the instantaneous frequency (a chirp) $\Delta \omega_{i}(t)=$ $-\partial \Phi_{i}(t) / \partial t$. This gives rise to spectral broadening or, equivalently, a reduction of the coherence time. Correspondingly, the radial variation of the intensity, $I(r)$, introduces a curvature of the phase front which, for large $\alpha$ or large $I$, will make the beam strongly divergent [15]. This is illustrated in Fig. 1 where we show the space- and time-dependent phase, and the wavelength variation induced, for a 100 fs (Gaussian) laser pulse with a wavelength of $810 \mathrm{~nm}$ and a peak intensity of $2 \times 10^{14} \mathrm{~W} / \mathrm{cm}^{2}$. In space the laser beam is a focused Gaussian, with a confocal parameter of $3 \mathrm{~mm}$. Note that the Gaussian beam itself has an $r$-dependent phase term. In Fig. 1(a), we show the $r$-dependent phase for $z=0$, where the Gaussian term is zero. The values of $\alpha_{i}$ have been chosen to be $\alpha_{1}=10^{-14} \mathrm{~cm}^{2} / \mathrm{W}$ and $\alpha_{2}=27 \times 10^{-14} \mathrm{~cm}^{2} / \mathrm{W}$, that - as we shall see in the following-are representative of the 15th harmonic in argon for this intensity range. These values are also very close to what is predicted from the SFA, for a harmonic belonging to the plateau region $[10,16]$.

Note how different both the curvature of the phase front and the chirp induced on the field are for the two components. This means that the fields generated by each of the two contributions will have different spatiotemporal characteristics. The field with the fast phase variations (large $\alpha$ ) will be much more divergent and spectrally broadened than the field with the slow phase variation. We can try to estimate the relative spectral widths and angular divergences of the two fields. If the spectral broadening were only due to the chirp coming from the intensity-dependent phase, we would expect a ratio between the spectral widths of

$$
\frac{\Delta \omega_{2}}{\Delta \omega_{1}}=\frac{\frac{\partial \Phi_{2}(t)}{\partial t}}{\frac{\partial \Phi_{1}(t)}{\partial t}}=\frac{\alpha_{2} \frac{\partial I(t)}{\partial t}}{\alpha_{1} \frac{\partial I(t)}{\partial t}}=\frac{\alpha_{2}}{\alpha_{1}}=27
$$
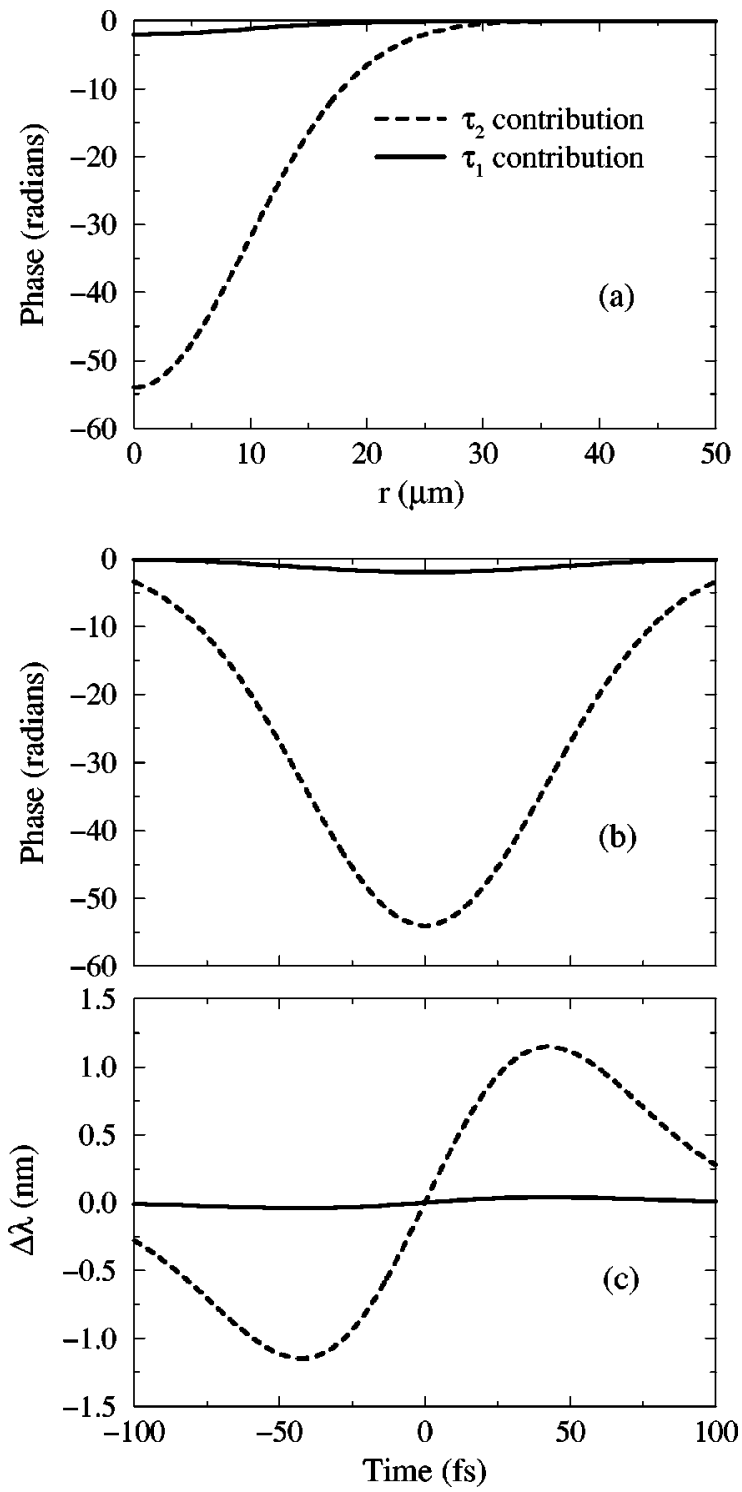

FIG. 1. We show the phase front (a) and the time-dependent phase (b) of two fields with phase behavior $\Phi_{1}(I)=-I$ (solid line) and $\Phi_{2}(I)=-27 I$ (dashed line). In (c) we show the chirp corresponding to the phase variation in (b).

and thus a ratio of $1 / 27$ between the coherence times, being inversely proportional to the spectral width. Equivalently, in a geometrical optics approximation, the relative angular divergence would be $\theta_{2} / \theta_{1}=\alpha_{2} / \alpha_{1}=27$.

For the $\tau_{2}$ field, the spectral width is mainly determined by the dipole chirp $\left(\Delta \omega_{2}=\alpha_{2} \partial I / \partial t\right)$. However, this is not the case for the $\tau_{1}$ contribution, for which the bandwidth obtained by assuming a Fourier-transform limited pulse is comparable to the (small) intensity-induced spectral broadening. This can be seen from Fig. 1(c). The spectral broadening induced by the dipole chirp can be estimated from the figure to be approximately $0.1 \mathrm{~nm}$ for the $\tau_{1}$ component and about $2 \mathrm{~nm}$ for the $\tau_{2}$ component. Assuming that the pulse length $T$ of the field of the 15th harmonic is between 20 and $50 \mathrm{fs}$ (in agreement with the measurements of [3]), one gets a transform limited bandwidth of the pulse of $\Delta \omega=2 \pi$ $\times 0.44 / T$, giving a $\Delta \lambda$ between 0.1 and $0.2 \mathrm{~nm}$. Thus, the spectrum of the $\tau_{2}$ field should be completely dominated by 
the dipole chirp, whereas the spectral width of the $\tau_{1}$ field should rather be determined by its Fourier transform (FT) limited broadening. We can, therefore, expect the ratio of the spectral widths to be smaller than 27, between 10 and 20. In the same way, the divergence of the $\tau_{2}$ field is mainly due to the effect of the (spatial) dipole chirp. For the $\tau_{1}$ contribution, the diffraction limited spreading is comparable to the spreading induced by the dipole phase. Consequently, this reduces the ratio of the divergence angles.

The total harmonic field is thus expected to macroscopically separate into its different quantum path components both in space (in the far field) and in time (in the spectrum). In the far field one should observe two regions, having different coherence times. The radiation in the inner regioncoming from quantum path 1 -should have a much longer coherence time than that in the outer region, coming from quantum path 2, in agreement with the experimental results from [1].

In the following section we test this idea, performing more elaborate numerical calculations of harmonic generation from atoms in strong fields. We perform a complete simulation of the harmonic generation process, including both the single atom response-calculated by numerical integration of the TDSE- and the macroscopic response. We also extend somewhat the simple model presented above, describing again the dipole moment as a sum of two fields with linear phase dependences. We find the values of the phase coefficients $\alpha_{i}$ and the weight of each quantum path by a phase analysis of the full quantum mechanically calculated dipole moment. This is done for all values of the intensity of the fundamental field, and the generated dipole moments are then used as a source of radiation in a propagation calculation.

\section{NUMERICAL CALCULATIONS}

\section{A. Method}

\section{Single atom data}

We start by calculating the response of one single atom to an intense linearly polarized laser field. This is done by numerical integration of the TDSE within the framework of the single active electron approximation, as described in [12]. The laser field is pulsed with a $\cos ^{2}$ envelope and a pulse length [full width at half maximum (FWHM)] of $\tau$. The emitted radiation spectrum is proportional to the Fourier transform of the acceleration $a(t)$ of the active electron, giving the dipole spectrum

$$
D(\omega)=\frac{1}{\tau} \frac{1}{\omega^{2}} \int_{-\infty}^{\infty} a(t) \exp (i \omega t) d t
$$

where $\tau$ is the pulselength, used as a normalizing factor, and the $1 / \omega^{2}$ term is due to the conversion between acceleration and length gauge.

We use an adiabatic approximation to extract the dipole data as function of the intensity of the driving field, as described in detail in [18]. We focus our attention onto some particular harmonic, $q \omega_{0}$. To calculate the time profile of that harmonic, the spectrum is multiplied by a window func- tion around the harmonic $F\left(\omega-q \omega_{0}\right)$ and inverse Fourier transformed into the time domain:

$$
D_{q}(t)=\frac{1}{N} \int_{-\infty}^{\infty} D(\omega) \exp (-i \omega t) F\left(\omega-q \omega_{0}\right) d \omega,
$$

where $N$ is a normalizing factor, proportional to the FWHM of the harmonic.

At every point in time $t$ we know the intensity of the driving field,

$$
I(t)=I_{0} \cos ^{2}\left(\frac{\pi}{2} \frac{t}{\tau}\right)
$$

where $\tau$ is the pulse length, and $I_{0}$ is the peak intensity. We now combine the knowledge of $D_{q}(t)$ and $I(t)$ to obtain the dipole strength and phase as function of the intensity $D_{q}(I)$ for intensities ranging from $I=0$ to $I=I_{0}$. By doing so we assume that the main features of the generation process are determined by the instantaneous intensity of the field, and not by the dynamics. In other words, we perform an adiabatic approximation. We consider only the first half of the pulse since, due to the influence of ionization, the time profile of the harmonic is not symmetric - the main part of the radiation being generated on the rising edge of the pulse.

\section{Quantum path analysis of $H H G$}

In order to analyze the dipole phase in terms of its quantum path components, we perform a numerical phase analysis as described in [17]. We describe the method only briefly here.

The dipole moment of some harmonic $q \omega_{0}$ as function of the intensity of the laser field has the following form:

$$
D_{q}(I)=A(I) \exp [i \Phi(I)]
$$

As we argued in the Introduction, the dipole moment consists of several contributions with phases of the form $\Phi_{i}(I)$ $=-\alpha_{i} I$. We wish to show that we can indeed separate the dipole moment into these contributions. In addition we want to extract information about the distribution of these phase components.

This problem is very similar to having a transient signal with several (frequency) components, of which one wants to make a spectral analysis [19]. In our case, the intensity $I$ plays the role of time, and the phase coefficient $\alpha$ corresponds to frequency. To compute the distribution of $\alpha$ 's (the "spectrum") for each intensity in a certain intensity range, we perform the equivalent of a time-frequency analysis. We thus multiply the numerically calculated dipole moment with a window function, maximized at some particular intensity $I=I_{m}$. The windowed dipole moment is then Fourier transformed, which yields a "spectrum" displaying one or several well separated peaks. ${ }^{1}$ These peaks correspond to quantum paths for the range of intensities for which the window function has large values, i.e., for intensities close to $I_{m}$. One can, therefore, assign the phase coefficients $\alpha_{i}$ deter-

\footnotetext{
${ }^{1}$ Actually, we let the dipole strength $A(I)$ be constant and analyze only the phase of the dipole moment
} 


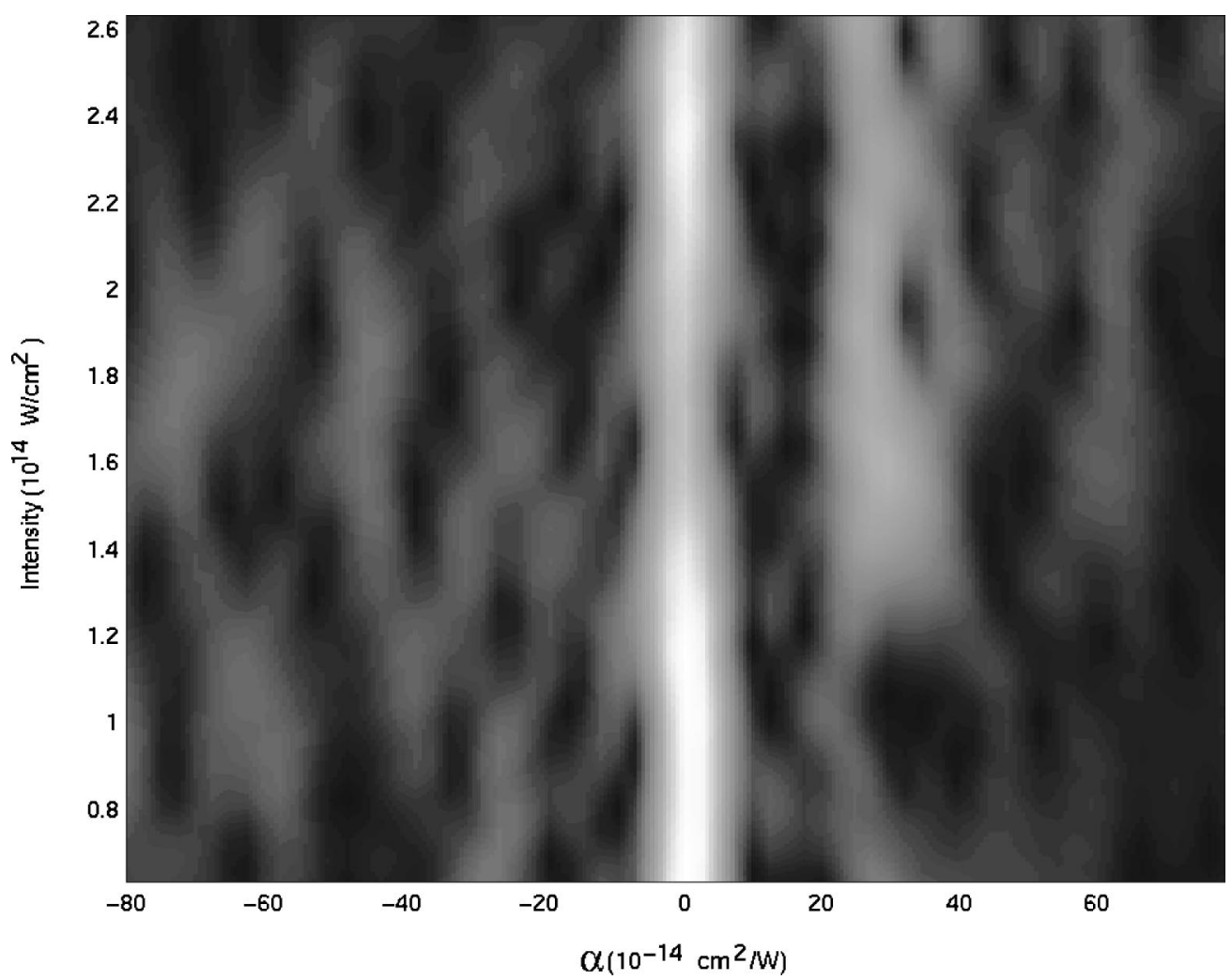

FIG. 2. We show in gray scale the distribution of $\alpha_{i}$ for each intensity of the fundamental.

mined from such a "spectrum" to the intensity $I_{m}$, keeping of course the uncertainty principle in mind: As in timefrequency analysis, the "spectral" resolution is determined by the width of the window function. This is then done for all values of the intensity $I_{m}$.

In Fig. 2 we show the result of such a quantum path analysis of the 15th harmonic in argon. The dipole moment as function of intensity has been calculated as described in the previous section, using results calculated within the TDSE approach with a peak intensity of $3 \times 10^{14} \mathrm{~W} / \mathrm{cm}^{2}$. In the figure, the abscissa is $\alpha$ in units of $10^{-14} \mathrm{~cm}^{2} / \mathrm{W}$, the ordinate is the intensity, in $10^{14} \mathrm{~W} / \mathrm{cm}^{2}$, and the distribution of $\alpha$ 's is shown in gray scale. Indeed, we find that there are mainly two peaks in each "spectrum," around $\alpha_{1} \approx 1$ and $\alpha_{2} \approx 27 \times 10^{-14} \mathrm{~cm}^{2} / \mathrm{W}$, and thus that the dipole moment can be written approximately as a sum of two contributions with linear phase behavior,

$$
D_{q}(I) \approx B_{1}(I) A_{1}(I) \exp \left(-i \alpha_{1} I\right)+B_{2}(I) A_{2}(I) \exp \left(-i \alpha_{2} I\right),
$$

$B_{i}(I)$ denoting the weight of each contribution, and $A_{i}(I)$ denoting the dipole amplitude. As the figure shows, the contribution $B_{1}$ of the slow phase behavior is somewhat larger than that of the fast phase behavior. Note that the linear phase dependence on the intensity has not been imposed on the dipole moment by using this method. The fact that we do find that the dipole moment-calculated by numerical integration of the TDSE - can largely be described by two contributions having a linear phase dependence is interesting in itself. The values of the $\alpha_{i}$ that we find correspond well to what is predicted from the SFA, whereas the relative weights of the two quantum paths differ somewhat; the SFA predicts the rapidly varying phase contribution to be more dominant in general.

The weight of each quantum path is calculated by integration over the FWHM of the $\alpha_{i}$ peaks, for each intensity $I_{m}$, and is normalized by the sum of the two weights. Having then obtained the phase coefficient and the weight of each quantum path component, we construct two sets of dipole data as function of intensity, each with a linearly decreasing phase, $\Phi_{i}=-\alpha_{i} I$. We use a strength $A_{1}(I)=A_{2}(I)$ for the two fields, which increases stepwise as a power law, where the appropriate powers have been found by curve fitting to the dipole data calculated in Sec. III A 1. The strengths are then multiplied by the weights calculated above.

\section{Propagation}

Having calculated the dipole strengths and phases, we proceed to propagate the $q$ th harmonic as described in [18], in the paraxial and slowly varying envelope approximations. We use the single atom dipole data-calculated either as in Sec. III A 1 or as in III A 2-as a source of radiation when solving the three-dimensional wave equation. We solve the propagation equation for each harmonic, of frequency $q \omega_{0}$, in cylindrical coordinates:

$$
\nabla_{\perp}^{2} E_{q}(r, z)+2 i k_{q} \frac{\partial E_{q}(r, z)}{\partial z}=-\frac{q^{2} k_{1}^{2}}{\epsilon_{0}} P_{q}(r, z) .
$$

In this equation $E_{q}(r, z)$ and $k_{q}$ denote the electric field and the wave vector, respectively, of the $q$ th harmonic. $P_{q}(r, z)$ is the polarization field of the harmonic, equal to the product of the atomic dipole moment and the atomic density. This is 
done for a number of points in time spanning the laser pulse, and we thereby obtain the space- and time-dependent field $E_{q}(r, t)$ at the exit of the medium. From this we calculate both the far-field and the spectral profiles.

The far field is calculated from the near-field profile by means of a Hankel transform [20],

$$
\begin{aligned}
E_{q}\left(r^{\prime}, z^{\prime}, t\right)= & -i k_{q} \int \frac{E_{q}(r, z, t)}{z^{\prime}} J_{0}\left(\frac{k_{q} r r^{\prime}}{z^{\prime}}\right) \\
& \times \exp \left(\frac{i k_{q}\left(r^{2}+r^{\prime 2}\right)}{2 z^{\prime}}\right) r d r,
\end{aligned}
$$

where $z^{\prime}$ is the (axial) distance from the exit of the medium to the observation plane, and $J_{0}$ is the zero-order Bessel function. The time-integrated intensity of the far field is

$$
I_{q}\left(r^{\prime}, z^{\prime}\right)=\int\left|E_{q}\left(r^{\prime}, z^{\prime}, t\right)\right|^{2} d t
$$

The spectrum is calculated by Fourier transforming the near-field profile and integrating over the transverse direction,

$$
I_{q}(\omega)=\int\left|\int_{-\infty}^{\infty} E_{q}(r, t) \exp \left[i\left(\omega-q \omega_{0}\right) t\right] d t\right|^{2} 2 \pi r d r .
$$

As mentioned above, ionization is included in the single atom calculation, which means that we do account for depletion, whereas dispersion effects of the free electrons in the medium are neglected (as well as other dispersion and absorption effects from atoms or ions).

\section{B. Numerical results}

In the following we show some calculations for the 15th harmonic in argon. We have chosen the parameters of the calculation so as to mimic approximately the experiments performed by Bellini et al. [1]. We use a Gaussian laser pulse with a pulse length of $100 \mathrm{fs}$, a peak intensity of 2 $\times 10^{14} \mathrm{~W} / \mathrm{cm}^{2}$, and a wavelength of $810 \mathrm{~nm}$. In space, the focused laser beam is taken to be a Gaussian, with a confocal parameter of $3 \mathrm{~mm}$ (thus, rather tightly focused). The nonlinear medium is a Lorentzian gas jet with a FWHM of 1 $\mathrm{mm}$.

We compare numerical results coming from the full calculation to results obtained using the two fields with linear phase dependences-calculated as in Secs. III A 1 and III A 2-that have been added coherently after propagation through the medium. In the experiment in [1], the far-field interference pattern of two harmonic sources separated by some distance was studied. In this way one could directly observe both the spatial separation of the two quantum path components, and-by delaying the two pulses relative to each other-the coherence time (i.e., the spectrum) in each of the regions. Here we have chosen to show the far-field pattern and the spectral profile separately, for reasons of clarity.

In Fig. 3(a) we show the far field of the 15th harmonic in argon. We show the result of the full calculation in a thick

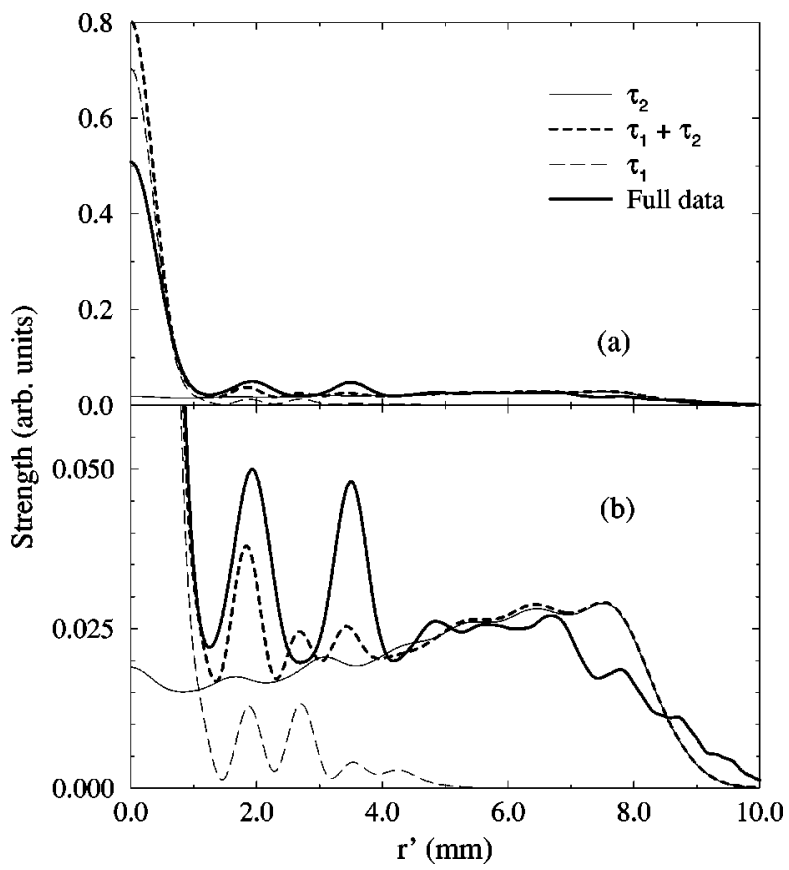

FIG. 3. We show the (propagated) far field of the 15th harmonic in argon. In the thick solid line is shown the result coming from propagation of the full set of data, in the thick dashed line the sum of the two quantum path fields, which are shown individually with thin lines. In (b) is shown an enlargement of the low intensity region in (a).

solid line, whereas the result of the sum of the two quantum path contributions is shown in a thick dashed line. The two individual contributions are shown in thin lines, solid for $\tau_{2}$ and long-dashed for $\tau_{1}$. In (b) an enlargement of the lowintensity region of (a) is presented.

There are several things to be learned from the figure. First, note how good the agreement is between the full data and the sum of the quantum path contributions, not only qualitatively, but even quantitatively. This means that it is certainly a good approximation to describe the harmonic field as consisting of two components with a simple, linear phase dependence. This in itself is a nice result since it can be utilized to qualitatively predict the outcome of experiments on harmonic generation. Second, when looking at the separate contributions of the two fields, we see as expected how the $\tau_{2}$ contribution is much wider than the $\tau_{1}$ contribution, the relative spreads being approximately 17, in agreement with the predictions from Sec. II.

In Fig. 4 we show the spectrum of the 15th harmonic in argon, calculated in the same conditions as above. Also here the agreement between the two models is striking. The harmonic radiation is clearly separated into two components having (very) different temporal coherence times. The ratio of the spectral widths is approximately 13, in good agreement with the above.

Let us note here the analogy between the space and time dependence of the harmonic radiation. In the near field as well as in time, the two quantum path contributions to the dipole moment do not separate, and, therefore, interfere. These interferences can be observed both in the space (near field) and in the time domain. The separation of the field into the two components appears in the spectrum and in the far 


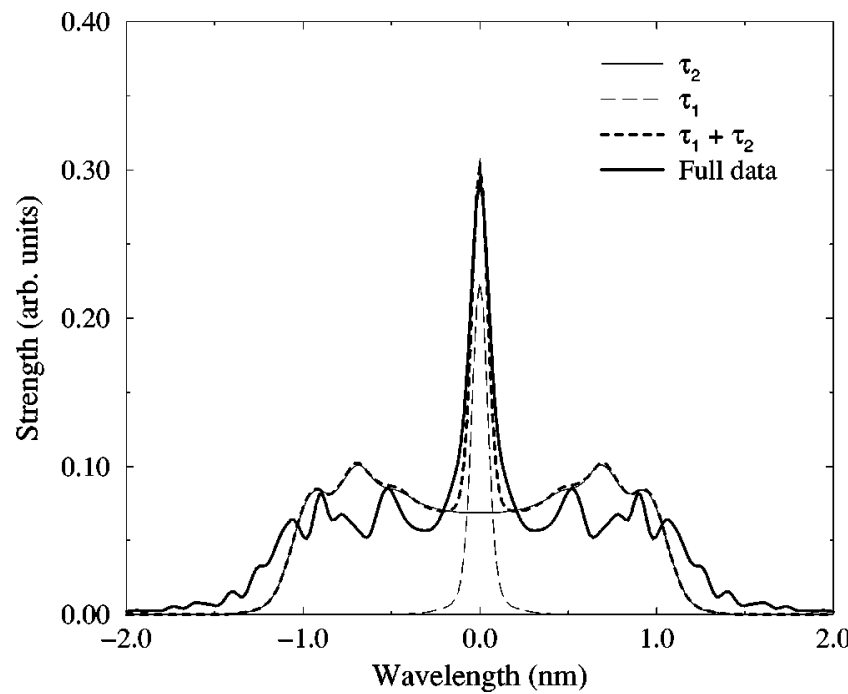

FIG. 4. The calculated spectrum of the 15th harmonic in argon. The parameters are as in Fig. 3.

field, since these are both related to the (time-dependent) near field by a Fourier (Hankel) transform (the Hankel transform is the equivalent of the Fourier transform, in two dimensions and expressed in cylindrical coordinates). The temporal and spatial coherence of the harmonic field are thus related, in the sense that they have the same origin, namely the variation of the phase with the intensity.

The modulations on the spectral profile, observed on the total field and the $\tau_{2}$ field, may be related to interference between radiation at some frequency generated at different times during the pulse, analogous to the interference pattern induced by self-phase modulation in a nonlinear medium [21]. Similar modulation can be seen on the far-field profile, and can be attributed to the same interference effect taking place in the spatial domain.

\section{Comparison with experimental results}

In [1] the far-field interference pattern between harmonic radiation generated in two sources, vertically separated in the gas jet by approximately $50 \mu \mathrm{m}$ was measured. Figure 3 of [1] illustrates the two different regions in the far field with different coherence times for the inner and outer region. The coherence times of the two regions were measured to be about 25 fs and 2.5 fs for the inner and outer regions, respectively [1], and the ratio between them is thus in good agreement with the theoretical predictions above. The relative spreads of the two regions is between 5 and 6 , which is slightly less than predicted by theory.

Peatross and Meyerhofer [13] also observed two spatial regions in the far-field profiles of harmonics in argon, krypton, and xenon, the far-field profiles exhibiting an intense inner region having broad shoulders.

Also experimental spectra exhibiting a narrow peak with low, broad shoulders have been observed, in this case for plateau harmonics in neon and argon [14].

\section{DISCUSSION}

We have analyzed the spatiotemporal behavior of harmonic radiation in terms of the quantum path contributions

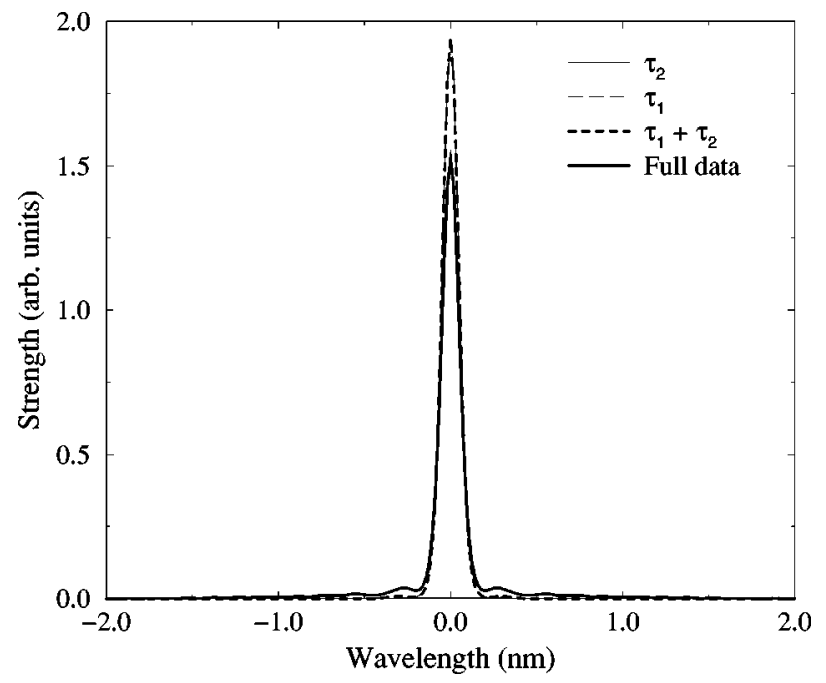

FIG. 5. The spectrum of the 15th harmonic in argon when the gas jet is placed $1.2 \mathrm{~mm}$ after the laser focus. The intensity (in the focus) is $2.6 \times 10^{14} \mathrm{~W} / \mathrm{cm}^{2}$.

to the harmonic dipole moment. We showed that due to the different phase dependence of the electronic trajectories, the harmonic field macroscopically separates both in the space and the spectral domain. This enables a direct(macroscopic) - study of the motion of the electronic wave packet in the continuum.

We showed that a simple model of harmonic generation, describing the harmonic field as a sum of two fields with linear phase dependence on the fundamental intensity, gives results in very good agreement with more elaborate numerical calculations, as well as with recent experimental results. This could make it easier to, at least qualitatively, predict the outcome of harmonic generation experiments.

An advantage of considering the two contributions to the harmonic field separately is that one can study the phasematching properties of each of the two fields. By finding the macroscopic/focusing conditions that best phase match one part of the field, and not the other, one can thus control the properties of the generated radiation. For instance, when the gas jet is placed a few mm after the laser focus, only the $\tau_{1}$ contribution survives phase matching [16]. This is illustrated in Fig. 5, where we show the spectrum of the 15th harmonic in argon, when the gas jet is placed $1.2 \mathrm{~mm}$ after the laser focus. The intensity in the focus is $2.6 \times 10^{14} \mathrm{~W} / \mathrm{cm}^{2}$. The $\tau_{1}$ contribution coincides with that of $\tau_{1}+\tau_{2}$. The $\tau_{2}$ contribution is nearly absent in this focusing condition (cannot be seen in the figure), which is in good agreement with the "true" harmonic profile shown in the thick solid line.

Finally, the spatial separation has an important consequence also related to control of the harmonic generation process. We remarked in Sec. III B that the spatial and temporal coherence properties are closely related, since they are both largely determined by the intensity dependent dipole phase. This means that by proper aperturing of the harmonic beam in the far field one can choose the inner region of the harmonic field, giving harmonic radiation with a large degree of both spatial and temporal coherence. This would be very 
useful for some applications of harmonic radiation. However, for other applications, the large divergence of the $\tau_{2}$ radiation may be useful, since it could mean that this part of the beam could be refocused to a smaller spot, and thus a higher intensity, than the part coming from the $\tau_{1}$ contribution [15]. Correspondingly, in time, the $\tau_{1}$ field gives rise to a temporally coherent pulse, whereas the $\tau_{2}$ part of the pulse, being strongly chirped, presents the possibility of being recompressed to a much shorter pulse.

\section{ACKNOWLEDGMENTS}

We thank M. Bellini, T. W. Hänsch, C. Lyngå, A. Tozzi, and C.-G. Wahlström for interesting discussions of the experimental results from [1]. This work was carried out in part under the auspices of the U.S. Department of Energy at the Lawrence Livermore National Laboratory under Contract No. W-7405-ENG-48. We gratefully acknowledge the support of the Swedish Natural Science Research Council.
[1] M. Bellini, C. Lyngå, A. Tozzi, M. B. Gaarde, T. W. Hänsch, A. L'Huillier, and C.-G. Wahlström, Phys. Rev. Lett. 81, 297 (1998).

[2] For a review, see A. L'Huillier, T. Auguste, Ph. Balcou, B. Carré, P. Salières, C. Altucci, M. B. Gaarde, J. Larsson, E. Mevel, T. Starczewski, S. Svanberg, C.-G. Wahlström, R. Zerne, K. S. Budil, T. Ditmire, and M. D. Perry, J. Nonlinear Opt. Phys. Mater. 4, 647 (1995).

[3] T. E. Glover, R. W. Schoenlein, A. H. Chin, and D. V. Shank, Phys. Rev. Lett. 76, 2468 (1996); A. Bouhal, R. Evans, P. Breger, R. C. Constantinescu, H. G. Muller, D. von der Linde, G. Grillon, A. Mysyrowicz, and P. Agostini, J. Opt. Soc. Am. B 14, 950 (1997).

[4] J. W. G. Tisch, D. D. Meyerhofer, T. Ditmire, N. Hay, M. B. Mason, and M. H. R. Hutchinson, Phys. Rev. Lett. 80, 1204 (1998).

[5] C. Lyngå, C. Delfin, M. Bellini, T. W. Hänsch, M. B. Gaarde, A. L'Huillier, and C.-G. Wahlström (unpublished).

[6] T. Ditmire et al., Phys. Rev. Lett. 77, 4756 (1996).

[7] P. B. Corkum, Phys. Rev. Lett. 71, 1994 (1993).

[8] K. J. Schafer, B. Yang, L. F. DiMauro, and K. C. Kulander, Phys. Rev. Lett. 70, 1599 (1993).

[9] W. Becker, S. Long, and J. K. McIver, Phys. Rev. A 50, 1540 (1994), and references therein.

[10] M. Lewenstein, Ph. Balcou, M. Yu. Ivanov, A. L'Huillier, and P. Corkum, Phys. Rev. A 49, 2117 (1994).
[11] M. Lewenstein, P. Salières, and A. L'Huillier, Phys. Rev. A 52, 4747 (1995).

[12] K. J. Schafer and K. C. Kulander, Phys. Rev. Lett. 78, 638 (1997).

[13] J. Peatross and D. D. Meyerhofer, Phys. Rev. A 51, R906 (1995).

[14] L. Roos and C. Delfin (private communication); also, L. Lederoff, B. Carré, and P. Salières (private communication).

[15] The spatiotemporal consequences of a rapidly varying dipole phase are discussed in detail in P. Salières, A. L'Huillier, P. Antoine, and M. Lewenstein, Adv. At., Mol., Opt. Phys. 41, 83 (1998).

[16] Ph. Antoine, A. L'Huillier, and M. Lewenstein, Phys. Rev. Lett. 77, 1234 (1996).

[17] Ph. Balcou, A. S. Dederichs, M. B. Gaarde, and A. L'Huillier (unpublished).

[18] M. B. Gaarde, Ph. Antoine, K. J. Schafer, K. C. Kulander, and A. L'Huillier, Phys. Rev. A 57, 4553 (1998).

[19] A. M. Marshall and F.-R. Verdun, Fourier Transforms in NMR, Optical and Mass Spectrometry-a User's Handbook (Elsevier, Amsterdam, 1990).

[20] A. L'Huillier, L.-A. Lompré, G. Mainfrey, and C. Manus, in Atoms in Intense Radiation Fields, edited by M. Gavrila (Academic Press, New York, 1992).

[21] Y. R. Shen, The Principles of Nonlinear Optics (Wiley, New York, 1984). 